\title{
Observation of Bending Wave Localization and Quasi Mobility Edge in Two Dimensions
}

\author{
Ling Ye, George Cody, Minyao Zhou, and Ping Sheng \\ Exxon Research \& Engineering Company, Route 22 East, Annandale, New Jersey 08801
}

Andrew N. Norris

Department of Mechanical \& Aerospace Engineering, Rutgers University, P.O. Box 909, Piscataway, New Jersey 08855

(Received 26 February 1992; revised manuscript received 11 May 1992)

\begin{abstract}
Localization of bending waves is observed on a steel plate decorated with Lucite blocks. A significant experimental feature of the localized modes is an exponential decay of the mode intensity from their peaked centers, with a decay length that increases as $\left(f_{0}-f\right)^{-1}$ when the mode frequency $f$ approaches a quasi mobility edge $f_{0}$. Our experimental results, together with finite-element calculations, support the mechanism of strong bending wave scattering by the Lucite block resonances as the source of the localization phenomenon.

PACS numbers: $63.50 .+\mathrm{x}, 03.40 .-\mathrm{t}, 43.40 .+\mathrm{s}, 63.30 .+\mathrm{d}$
\end{abstract}

The concept of wave localization was first proposed by Anderson in the context of electronic transport in disordered materials $[1,2]$. Over the past three decades, the importance of the localization concept in the physics of electronic transport and the metal-insulator transition has been extensively documented [3]. However, as a phenomenon in itself, Anderson localization has eluded direct observation in electronic systems. This is due to both the difficulty in mapping electronic wave functions, as well as the difficulty in isolating the Anderson localization from the ever-present electron-electron interaction and the consequent many-body effects. The fact that such intrinsic difficulties are absent in classical wave systems is one of the reasons that the study of elastic-wave and light localization has been a topic of active pursuit in recent years [4-6].

In this work, we report the direct observation of localized elastic waves in a composite two-dimensional system-the bending wave of a steel plate decorated with Lucite blocks. The localized mode is characterized by an exponentially decaying intensity envelope away from the center. Measurement of the frequency dependence of the decay length yields evidence for a "quasi mobility edge" which separates the strongly localized modes from the more extended modes, with the decay length increasing as $\left(f_{0}-f\right)^{-1}$ when the mode frequency $f$ approaches the quasi mobility edge frequency $f_{0}$. Our results support the mechanism of strong bending wave scattering by the shear resonances of the Lucite blocks as the source for the observed localization phenomenon.

The experimental system consists of a $1.83-\mathrm{m} \times 1.83$ $\mathrm{m} \times 2.54-\mathrm{cm}$ steel plate set on four vibration-isolation supports. The excitation source used in the experiment is a $B \& K$ force transducer, which produces a pulse 0.025$0.04 \mathrm{~ms}$ in duration in an area less than $1 \mathrm{~mm}^{2}$. The frequency content of the pulse is flat within $10 \%$ from 0 to 6 $\mathrm{kHz}$. The detector is a $\mathrm{B} \& \mathrm{~K}$ accelerometer, which is magnetically attached to the plate and measures the local vertical acceleration. The signals from both the force transducer and the accelerometer are analyzed with a dual-channel signal processor, whose output is the transfer function $H(f)=\left\langle a(f) F^{*}(f)\right\rangle /\left\langle F(f) F^{*}(f)\right\rangle$, where $a(f)$ and $F(f)$ denote the Fourier transforms of the measured acceleration and excitation force, respectively, the asterisk denotes complex conjugation, and the angular brackets denote averaging over repeated excitationdetection measurements under the same condition. $H(f)$ expresses the acceleration response of the system as a function of frequency for a given source-receiver configuration. The averaging process is important for the reduction of electronic noise and interference with transient signals from edge reflections.

The excitations of an elastic plate [7] can generally be divided into two frequency regimes. Excitations in the plate thickness direction have a discrete spectrum, with a nonzero minimum frequency given by (shear wave speed) $/ 2 d \approx 65 \mathrm{kHz}$. For $f \leq 65 \mathrm{kHz}$ the excitation modes may be regarded as two dimensional, both in the sense that the wavelength $>d$ as well as in the sense that the wave transport occurs only in the two directions parallel to the plate. In the present case, the frequency range of interest is from 0 to $6 \mathrm{kHz}$, and only the bending wave, the displacement of which is transverse in nature and perpendicular to the plate, is excited by the impact source as well as measured by the accelerometer. Comparison of the experimental spatial intensity distribution of the plate excitation at $1.5 \mathrm{kHz}$ with bending wave calculations [8] shows excellent quantitative agreement, including the separation between the intensity maxima of $39 \mathrm{~cm}$.

Two hundred $8.89-\mathrm{cm} \times 8.89-\mathrm{cm} \times 7.62-\mathrm{cm}$ Lucite blocks are solidly bonded to the steel plate in either a periodic, checkerboard pattern or a random arrangement. The solid bond between the Lucite and steel is made by locally heating the plate above the melting point of salol [9]. The bonding has a nonuniform contact (because salol does not wet Lucite when it is in the liquid state), which is visible through the transparent Lucite blocks. 
Thus, even the periodic arrangement of blocks has significant randomness in the steel-Lucite contacts.

Measurements of the composite-system excitations are carried out with two types of source-receiver configurations on the side of the steel plate opposite to the bonded Lucite blocks. The first type of measurement configuration (type I) is the point-source-point-detector arrangement. The second type (type II) employs the coherent addition of the point-source excitations along a line, with detectors also placed along a line parallel to the excitation line. Type II measurements generally show much less fluctuation than the type I measurement due to the decreased sensitivity to noise.

Figure 1(a) displays the frequency spectrum of the composite-system displacement velocity [defined as $|H(f)|^{2} / f^{2}$ ] in logarithmic scale when the Lucite blocks are arranged in the periodic configuration, measured with the type II configuration and with a source and detector line separation of $160 \mathrm{~cm}$. The most striking frequency regime of Fig. 1 (a) is that of $2 \mathrm{kHz}<f<3.5 \mathrm{kHz}$, where the square of the transfer function is 2 to 3 orders of magnitude smaller than the response in the other two re-

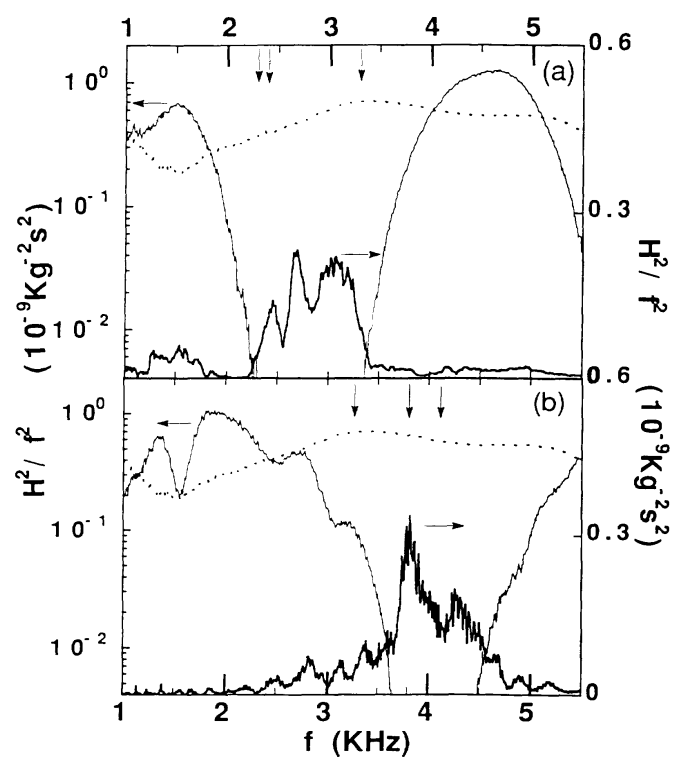

FIG. 1. The square of the velocity transfer function, expressed as $H^{2}(f) / f^{2}$, for the line-source-line-detector configuration at a separation of $160 \mathrm{~cm}$. $H(f)$ is obtained from time domain data filtered by a $3.5-\mathrm{ms}$ time window to eliminate residual edge reflections. The measured bare-steel-plate response is also shown in dashed line for comparison. The scale of the velocity transfer function is defined by the left-hand-side logarithmic coordinate. Two hundred Lucite blocks are glued on the steel plate in a checkerboard pattern. (a) The Lucite blocks are $8.89 \mathrm{~cm} \times 8.89 \mathrm{~cm}$ and $7.62 \mathrm{~cm}$; (b) the Lucite blocks are $8.89 \mathrm{~cm} \times 8.89 \mathrm{~cm}$ and $6.35 \mathrm{~cm}$. The thick solid lines in both (a) and (b), whose scale is defined by the right-hand-side linear coordinate, denote the square of the transverse velocity transfer function of a Lucite block. The vertical arrows indicate the positions of the calculated shear resonance frequencies. gimes. The same frequency gap is found when the source-detector lines are rotated $45^{\circ}$. The gap is thus isotropic.

The spatial intensity distributions of the modes at two different frequencies inside the gap are shown in Fig. 2. The mode distributions were mapped with a fine mesh of detector locations in the type I configuration and for a fixed source location at the plate center. The wave modes within the gap region $(2-3.5 \mathrm{kHz})$ are clearly localized as shown, with each of the localized modes characterized by a high peak, the position of which is independent of the source position (as long as the source remains inside the spatial domain where the mode has significant amplitude), with an isotropic exponentially decaying envelope.

Localized modes should be insensitive to the conditions outside their localization lengths. This condition was verified in a separate experiment by removing successive layers of Lucite blocks centered at the intensity peak of a localized mode. It is found that the mode at $f=2.8 \mathrm{kHz}$ does not alter its configuration at all up to the removal of the third layer from the center. However, with the removal of the third layer the intensity peak drops abruptly (by $40 \%$ ), together with the appearance of bending wave amplitude everywhere on the plate. This last observation

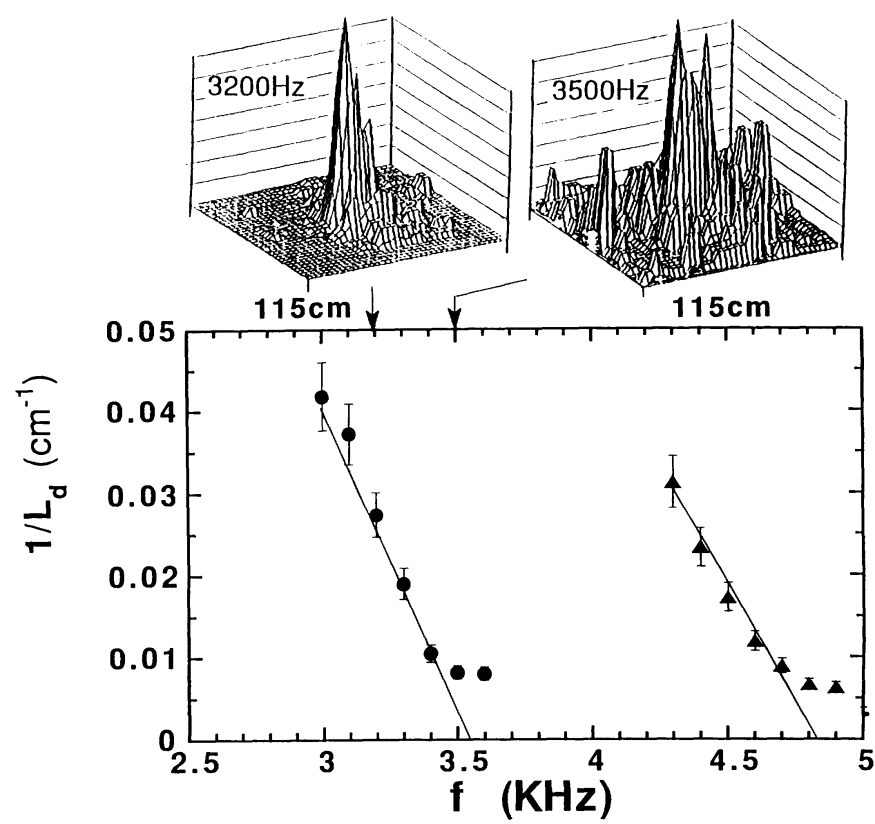

FIG. 2. The inverse exponential decay length, $1 / L_{d}$, for the amplitude plotted as a function of frequency for the two cases shown in Fig. 1. The decay length is measured by the type II configuration through the variation of the source-detector separation. The solid lines are the linear fits given by $1 / L_{d}$ $=a^{-1}\left(f-f_{0}\right)$, where $f_{0}=3.5$ and $4.8 \mathrm{kHz}, a=13$ and $18 \mathrm{~cm}$, and $f$ is in units of $\mathrm{kHz}$. The spatial distributions of the modes for the 7.62-cm-high Lucite blocks are shown at 3.2 and 3.5 $\mathrm{kHz}$. Symmetry with respect to the gap center has been verified for the 7.62-cm-high block case, with a lower mobility edge at $2.2 \mathrm{kHz}$. 
can be understood in terms of wave interference resulting from scattering between the blocks. Indeed, no localization can be found when only a single block is present on the steel plate.

It may be tempting to associate the frequency gap seen in Fig. 1(a) with coherent scattering due to periodicity. However, this association is easily shown to be false. The frequency response of the system, where the Lucite blocks are randomly arranged, is found to exhibit the same frequency gap as that seen in Fig. 1 (a) for a periodic array. Inside the frequency gap of the random array, localized modes are found just as in the periodic case. However, the profiles of the localized modes are more varied, with some exhibiting multiple intensity peaks. The presence of the frequency gap in both periodic and random configurations suggests that the randomness introduced into the periodic case at the Lucite-steel interfaces (due to the bonding material) is already sufficient to localize the bending wave.

In Fig. 1(a) the curve whose scale is defined by the right-hand-side linear coordinate denotes the measured transverse displacement velocity of a Lucite block on the steel plate when it is excited by a point impact on the steel plate and the detector is placed on the side of a Lucite block. The three response peaks at 2.4, 2.6, and 3.1 $\mathrm{kHz}$ coincide remarkably well with the position of the observed frequency gap and are also in excellent agreement with the three calculated shear resonance frequencies (shown by vertical arrows) using the finite-element method as described below. The width of the observed shear resonance modes also agrees well with the measured lifetime of the localized modes of $\sim 2 \mathrm{~ms}$.

To further verify the connection between the Lucite block shear resonances and the localized states, we have repeated all the experiments with 200 Lucite blocks with only $\frac{5}{6}$ the original height so that the measured shear resonances are shifted to about $3.5-4.5 \mathrm{kHz}$. Figure 1 (b) gives the results. As expected, the frequency response gap is now at higher frequencies. From the fact that here, again, the measured Lucite resonances fall right in the observed gap, we conclude that resonant scattering by Lucite blocks is responsible for the observed localization phenomenon. However, we note that for reasons unknown the lowest calculated block resonance is not a prominent feature of the measured response, but the higher-frequency resonance peaks are noted to be in reasonably good agreement with the calculated frequency positions, indicated by arrows.

The transition between the localized and the more extended states in a two-dimensional disordered system is a question of considerable interest $[5,10-12]$. It has been conjectured that there could be "quasi mobility edge(s)" in two-dimensional disordered systems [10,11,13] which separate the strongly localized states from those whose localization lengths may be transcendentally large [14], or even power-law localized. We have measured the ex- ponential decay length of the amplitude as a function of frequency in both source-detector configurations, and found it to vary as $|H|=\left|H_{0}\right| e^{-r / L_{d}}$ and $|H|=\left|H_{0}\right|$ $\times e^{-r / L_{d}} / \sqrt{r}$ for the type II and type I measurement configurations, respectively. Measurement of $L_{d}$ shows that it increases rather rapidly as the edge of the frequency gap is approached. In Fig. 2, the inverse of the measured amplitude decay length, $1 / L_{d}$, is plotted as a function of frequency for the upper halves of the frequency gaps shown in Figs. 1 (a) and 1(b). The error bars are estimated from the spatial resolution in the measurement of a fine mesh of detector locations. A linear relationship is found up to $L_{d} \sim 100 \mathrm{~cm}$, which is about half the size of the system. A linear fit to the data yields $L_{d} \approx a\left(f_{0}\right.$ $-f)^{-1} \mathrm{~cm}$, where $f$ and $f_{0}$ are expressed in $\mathrm{kHz}, a=13$ and 18 , and $f_{0}=3.5$ and 4.8 for the two cases shown in Figs. 1(a) and 1(b). This behavior suggests a quasi mobility edge at $f=f_{0}$. Furthermore, coincidence of the upper end of the Lucite shear resonance spectrum, 3.5 and $4.8 \mathrm{kHz}$, with the quasi mobility edge $f_{0}$ suggests that the physics of the quasi mobility edge lies in the change of the localization mechanism from resonant to nonresonant scattering below and above $f_{0}$. Since at a fixed measurement distance we have $\log \left(H^{2} / f^{2}\right)$ $\sim-1 / L_{d}(f)$, the frequency dependence of $L_{d}$ directly translates into the frequency dependence of the $H^{2} / f^{2}$ gap. Therefore, the observed gap symmetry in Fig. 1 implies similar behavior for the lower halves of the gaps. As a result of the finite size of the system, the nature of the states for $f>f_{0}$ is impossible to ascertain in the present experiment.

The role of resonant scattering in enhancing localization has been the prediction of numerous theoretical calculations [2,15]. To explore this effect for Lucite blocks bonded periodically on an infinite steel plate with a checkerboard pattern, a finite-element calculation was carried out by dividing a single Lucite block into $8 \times 8$ $\times 12$ elements, with traction-free boundary conditions on five sides and the sixth side joined on to the steel plate, which is divided into $16 \times 16 \times 4$ elements within the unit cell. The block boundary condition is applied at four sides of the unit cell. Young's moduli of $4 \times 10^{9}$ and $2.07 \times 10^{11} \mathrm{~N} / \mathrm{m}^{2}$, Poisson's ratios of 0.4 and 0.286 , and densities of 1.18 and $7.84 \mathrm{~g} / \mathrm{cm}^{3}$ were taken for Lucite and steel, respectively. The results for the 2.5 -in.-high Lucite blocks are shown in Fig. 3 for the [11] wave propagation direction. The following points should be noted: (1) The periodicity-induced gap at the block wave vector $k=25.3 \mathrm{~cm}^{-1}$ is not only small (less than $100 \mathrm{~Hz}$ ), but also shows directionality and occurs at the wrong frequency. The periodicity effect as the cause of the observed gap can thus be ruled out. (2) The three resonance modes introduced by Lucite blocks are evident at $k=0$ with frequencies at $3.26,3.65$, and $4.12 \mathrm{kHz}$. These modes have nearly flat dispersions, indicating a low group velocity that makes them waiting to be localized. 


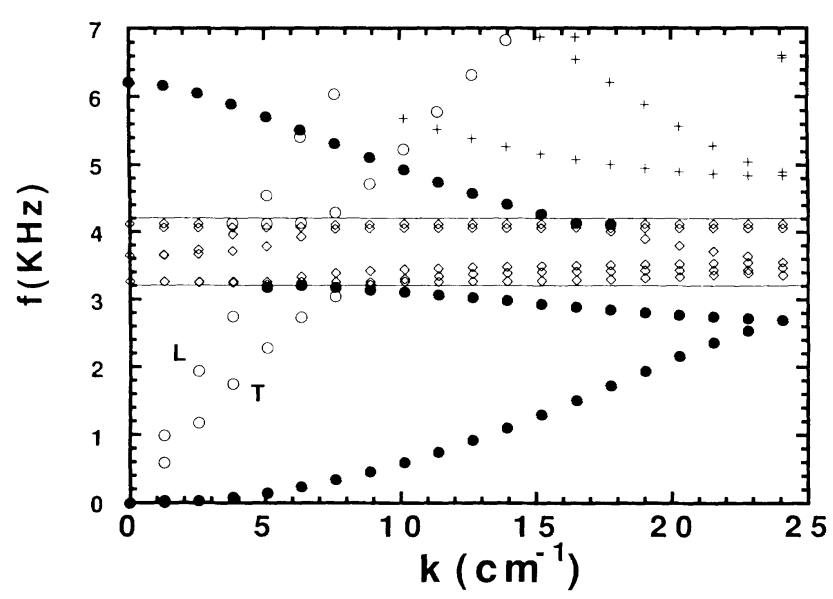

FIG. 3. Dispersion relations of the excitations for an infinite steel plate decorated with $8.89 \times 8.89 \times 6.35-\mathrm{cm}$ Lucite blocks in checkerboard pattern. The block wave vector $k$ is along the [11] direction. The modes noted as $T$ and $L$ involve only displacements parallel to the steel plate. They are not excited or detected in the present experiments. The bending wave is denoted by solid circles. The solid horizontal lines delineate the gap region. The symbols inside denote Lucite resonance modes. The crosses denote bending waves from higher Brillouin zones.

The high-frequency Lucite resonance is predominantly a shear resonance whose frequency is lowered and whose characteristics are modified by coupling to the steel plate; e.g., its frequency no longer varies strictly as the inverse of block height $h$, and it also acquires a slight vertical displacement. On the other hand, the two lower modes have predominantly flexural characteristics [16]. All three modes exhibit vertical displacement that is $\sim 10 \%$ of the overall horizontal displacement. At the Lucite-steel interface, however, the vertical displacement, which is responsible for coupling to the bending wave, is about 8 to 10 times the small horizontal displacement. The two lower-frequency resonances differ slightly in the distribution of vertical displacement in the Lucite and the steel plate, and we believe that the coupling to the steel is the source of their split in frequencies. Since the position of the gap varies with the block height, the present phenomenon is potentially useful as a frequency-tunable attenuation mechanism for bending waves propagating in structural walls. Experimental exploration of these issues is presently underway.

[1] P. W. Anderson, Phys. Rev. 109, 1492 (1958).

[2] Scattering and Localization of Classical Waves in Random Media, edited by P. Sheng (World Scientific, Singapore, 1990).

[3] P. A. Lee and T. V. Ramakrishnan, Rev. Mod. Phys. 57, 287 (1985).

[4] S. He and J. D. Maynard, Phys. Rev. Lett. 57, 3171 (1986).

[5] R. L. Weaver, Wave Motion 12, 129 (1990).

[6] For light localization, see B. Levi, Phys. Today 44, No. 6, 17 (1991), and references therein.

[7] R. L. Weaver and Y. H. Pao, J. Appl. Mech. 49, 821 (1982); for detailed derivation of the bending wave equation, please see L. Cremer and M. Heckl, StructureBorne Sound (Springer-Verlag, Berlin, 1988), in which some effects of a blocking mass on bending waves have been considered in a $1 \mathrm{D}$ geometry.

[8] L. D. Landau and E. M. Lifshitz, Theory of Elasticity (Pergamon, New York, 1986), 3rd ed., p. 103. The solution with a changed boundary condition is used.

[9] Salol is a short name for the chemical compound phenyl salicylate. Its melting point is $45^{\circ} \mathrm{C}$.

[10] Y. Meir, A. Aharony, and A. B. Harris, Europhys. Lett. 10, 275 (1989).

[11] M. Kaveh, Philos. Mag. B 52, 521 (1985).

[12] C. M. Soukoulis and G. S. Grest, Phys. Rev. B 44, 4685 (1991).

[13] The Koslowski and W. von Niessen, Phys. Rev. B 42, 10342 (1990).

[14] In the weak-scattering limit, the coherent backscattering effect in two dimensions increases logarithmically as a function of sample size. This implies an exponentially large localization length.

[15] The effect of resonant scattering on localization is discussed in P. Sheng and Z. Q. Zhang, Phys. Rev. Lett. 57, 1879 (1986).

[16] L. D. Landau and E. M. Lifshitz, Theory of Elasticity (Ref. [8]), p. 101. As a result of the fact that the crosssectional dimension of the Lucite block is comparable to its height, the frequencies of two lower-frequency resonances are significantly lowered compared to that predicted for thin rods. This fact is, and has been, verified by finite-element calculations. 